\title{
Les émergences en recherche-action : sérendipité ou choc de culture?
}

Emmanuel Legrand

\section{(2) OpenEdition \\ Journals}

Édition électronique

URL : http://journals.openedition.org/ere/5161

DOI : $10.4000 /$ ere. 5161

ISSN : 2561-2271

Éditeur

Centr'ERE

Référence électronique

Emmanuel Legrand, "Les émergences en recherche-action : sérendipité ou choc de culture? », Éducation relative à l'environnement [En ligne], Volume 4 | 2003, mis en ligne le 14 septembre 2003 consulté le 18 juin 2020. URL : http://journals.openedition.org/ere/5161 ; DOI : https://doi.org/ 10.4000/ere.5161 


\section{Les émergences en recherche- action : sérendipité ou choc de culture?}

Emmanuel Legrand

\section{Quelle culture, quel développement?}

1 S'il existe plusieurs acceptions du terme « culture », la tradition humaniste occidentale privilégie une vision de la culture qui se réduit à la philosophie et aux arts (surtout aux beaux-arts). Une orientation qui émerge depuis plusieurs années à travers, entre autres, l'anthropologie, la sociologie et la sociopsychologie adopte plutôt l'acception suivante: la culture est cette partie de notre identité que nous avons acquise et acquérons par le fait de naître et de vivre dans une société déterminée. Cette société, par son organisation, sa langue, sa vision du monde (au sens de cosmogonie), etc. est un des facteurs structurants de notre personnalité ou, tout au moins, de nos modes de fonctionnement ${ }^{1}$.

2 Cette conception de la culture fait souvent écho à une vision « ethnique » du terme. On peut toutefois envisager le cas des (sous-) cultures socioprofessionnelles, donnant lieu entre autres à diverses conceptions du développement (personnel, professionnel, social) au sein des divers membres d'une même société. Il suffit de conférer à la formation académique ou aux milieux de travail un même pouvoir structurant (incluant ses limites) sur les façons de penser et les modes de fonctionnement que celui que l'on attribue à la société d'origine.

Cependant, il paraît important de ne pas attribuer un fonctionnement mécanique et une efficacité absolue ${ }^{2}$ au processus "d'enculturation » ${ }^{3}$. Le fait même d'être capable de se rendre compte de l'existence de diverses cultures (ne dit-on pas que les voyages forment la jeunesse) montre que jusqu'à un certain point, la culture est médiatisée par nos capacités métacognitives. Concrètement, la rencontre d'une personne d'une autre culture (professionnelle par exemple) peut-être l'occasion de nous interroger sur nos 
propres modes de penser et de fonctionner et de les améliorer dans un processus de réflexion critique. Il est ici question d'autodéveloppement mutuel par confrontation culturelle.

4 Dans la pratique d'un chercheur en formation à l'ErE en milieu scolaire, il y a un lieu par excellence où le «choc culturel» est possible, c'est lorsque nous pratiquons la recherche-action. En effet, la recherche-action dans le cadre de la formation en ErE met généralement en présence des chercheurs d'un côté et des enseignants de l'autre, avec les différences de fonctionnement et de points de vue inhérents à ces deux cultures socioprofessionnelles différentes. Certes, cela peut paraître «évident»; mais la vision réflexive-critique est susceptible toutefois de jeter un nouveau regard sur cette question des cultures socioprofessionnelles dans le cadre de recherches-actions.

5 En effet, il n'est pas rare que lors de recherches (qu'il s'agisse de recherches-actions ou autres), on met en évidence d'autres phénomènes, d'autres variables que celles qui étaient prévues à l'origine. La découverte d'éléments inattendus a été désignée par le terme de "sérendipité " , de l'anglais serendipity, signifiant « découverte heureuse et inattendue ». Ces éléments nouveaux et imprévus ont reçu également l'appellation "émergences " au sens où ces éléments sont considérés comme déstructurant le modèle initial $d u$ chercheur et lui permettant de reconstruire un autre de niveau supérieur dans le cadre de la vision réflexive-critique, plus souvent attachée à la recherche-action. Cette question de l'émergence est d'ailleurs un point central de la vision « réflexive-critique », puisque cette vision insiste sur la réflexion et la critique de ses propres modèles.

6 Là où la question du choc culturel intervient, c'est dans le fait que, dans une vision réflexive-critique, on ne peut plus continuer à concevoir ces "émergences déstructurantes et restructurantes » de la recherche-action comme le fruit du hasard seulement (comme le suggère une vision positiviste de la recherche), mais plutôt comme le fruit même de la confrontation de cultures socioprofessionnelles en présence. Cela donne à la vision réflexive-critique son plein sens de critique sociale, parce que chaque partenaire peut faire consciemment la démarche de clarifier/ négocier non pas seulement un point d'intersection admissible entre les deux cultures socioprofessionnelles, mais la reconstruction d'une vision nouvelle qui englobe les points de vue initiaux. La prise en compte des émergences en recherche-action pourrait-elle devenir un acte volontaire, voire programmé ?

7 Maintenant que sont délimités les aspects de la culture (sous-culture socioprofessionnelle), du développement (autodéveloppement mutuel endogène) et de l'ErE (formation en ErE par la recherche-action) qui sont au centre de cet article, nous allons situer le contexte d'une recherche-action que nous avons menée et qui a donné lieu à des résultats inattendus et féconds. Nous présenterons une première émergence qui servira à mettre en évidence le travail de réflexion critique sur les modes de fonctionnement hérités des cultures socioprofessionnelles et à en monter la portée "développementale». Une seconde émergence servira à appuyer ce potentiel «développemental» avant d'introduire, in fine, les démarches mises en place pour favoriser la prise en compte des émergences dans un cadre interprétatif qui dépasse l'acception positiviste du concept de sérendipité. 


\section{Le contexte : une recherche-action} école, et un pédagogue, spécialisé en $\mathrm{ErE}$ de la Fondation Universitaire Luxembourgeoise (FUL) se sont réunis afin de mettre en place une formation initiale en ErE pour les futurs professeurs du secondaire inférieur. propres à l'équipe, sur la base d'une vision commune concernant l'environnement et l'éducation, ainsi que du rapport entre les deux :

- Pour les étudiants de première année, il était prévu de mettre en place une formation axée sur la résolution réelle de problèmes environnementaux locaux. Nous nous situions principalement dans une approche résolutique. Il ne s'agissait pas seulement toutefois d'une simple « exercisation » sur la capacité à résoudre des problèmes dans une vision positiviste, mais d'intégrer à la démarche de résolution de problèmes une vision systémique qui tienne compte entre autres des valeurs et des croyances.

- La formation en première année devait aussi se faire en ayant recours à la pédagogie active, en particulier la pédagogie du projet. Nous partions du principe qu'il fallait que ces futurs professeurs vivent eux-mêmes, lors de leur formation initiale, ces démarches pédagogiques qui étaient prônées en ErE. En effet, il y avait de fortes présomptions sur le fait que plus de la moitié d'entre eux n'en avaient quasi jamais vécu lors de leurs parcours scolaires.

10 Nous n'avions pas prévu de donner des cours théoriques sur les pédagogies actives, mais tout simplement de prendre le temps après chaque activité pour un moment de métacognition avec les étudiants sur la démarche pédagogique vécue. En principe, les aspects théoriques liés aux pédagogies actives seraient, de toute façon, travaillés lors des années de formation suivantes puisqu'ils s'agissaient d'étudiants de première année.

11 Après cette phase de définition commune, les professeurs et le chercheur ont collaboré à parts égales dans la conception, la préparation, la mise en place et l'évaluation des activités d'ErE proposées aux étudiants, futurs professeurs. Cela sous-entend évidemment un grand nombre de réunions de préparation et d'évaluation. Cette collaboration a permis aux professeurs, formateurs de professeurs, d'entrer eux-mêmes dans un processus d'autoformation par la pratique de la réflexion critique sur leurs propres actes de formation.

12 À ce volet "action » correspondait un volet « recherche » dont les objectifs étaient d'identifier les facteurs favorables et les facteurs limitants pour la mise en place de ce type de formation au sein d'un ISP : support ou problèmes institutionnels, avantages ou difficultés de la collaboration pour l'interdisciplinarité et du partenariat avec des personnes-ressources extérieures, etc.

Si la recherche a permis de mettre en évidence certains des facteurs recherchés, elle a permis aussi, par le biais de la clarification, de la discussion critique et de la négociation sur les différences de fonctionnement entre les participants (rattachés à différentes cultures socioprofessionnelles), l'apparition d'une «émergence » concernant la didactique. Peu à peu, les conceptions respectives ont été révisées et un nouveau modèle commun a été construit, tâche qui n'était absolument pas prévue au départ. 


\section{Une première « émergence »}

voulions aussi adopter une pédagogie active. Mais cette fois, l'équipe en place se trouvait devant une difficulté, elle n'avait jamais travaillé ensemble sur d'autres pédagogies actives que la pédagogie de projet. La différence de formation pédagogique et scientifique des protagonistes était suffisamment grande pour que les conceptions de la pédagogie active soient différentes. La clarification de nos conceptions a permis de mettre en évidence les différences et les ressemblances suivantes dans nos visions respectives :

- Pour les professeurs, la pédagogie active se modulait autour de la question de «l'articulation » entre théorie et exercices. Ces derniers étant principalement vus comme des exercices actifs et concrets, dans le même sens qu'un "scientifique » va sur « le terrain » ou en «labo ", par opposition à un travail livresque. Les liens qui pouvaient unir «théorie » et «exercice» étaient définis par l'ordre dans lequel ils étaient effectués. Un travail théorique suivi d'exercices était considéré comme une méthode déductive, un travail pratique suivi d'une théorie comme une méthode inductive, selon le modèle des mathématiques, reprenant ainsi le rapport qui unit les faits et la réalité lors de la construction de la connaissance scientifico-mathématique : les faits servent à construire des théories et ces théories permettent de prédire les faits.

- Pour le pédagogue, le point central de la pédagogie active était le constructivisme ${ }^{6}$, pris dans le sens simple que le travail pratique proposé aux étudiants devait permettre de reconstruire la théorie. Un autre point essentiel à atteindre était que cette reconstruction de la théorie ne devait pas rendre les étudiants seulement capables d'utiliser la théorie sur les cas utilisés pour le découvrir, mais aussi de le transférer à d'autres situations similaires (et donc de rendre les étudiants capables de repérer eux-mêmes le fait qu'ils soient dans une situation similaire).

Un point commun unissait ces professionnels, le refus de fonctionner seulement avec des exposés théoriques avec comme idées sous-jacentes :

1. qu'un cours théorique n'est que trop souvent mal compris par les étudiants et peu motivant ;

2. qu'un cours théorique risque de produire des étudiants inaptes à agir en situation réelle parce qu'ils ne reconnaîtront pas forcément le fait qu'ils sont dans la situation concrète qui concerne la théorie apprise ;

3. qu'un cours théorique n'aboutit que trop souvent à un niveau d'apprentissage et d'évaluation ne dépassant pas les objectifs de reproduction (l'apprentissage par cœur);

4. qu'il ne suffit pas toujours de donner oralement des exemples où la théorie s'applique et qu'il est préférable de la faire réellement appliquer.

D'un côté, la vision en induction/déduction (I/D) n'est pas nécessairement du constructivisme (ni pour certains de la pédagogie active), car elle conserve un aspect transmissif dans la mesure où le savoir est toujours, au début ou à la fin, donné par le professeur à partir d'une théorie préexistante. De l'autre côté, dans la vision 
constructiviste, la capacité des étudiants à reconstruire certaines théories difficiles n'est pas garantie non plus. L'approche induction/déduction est plus rassurante, mais elle risque d'avoir une moins grande efficacité en termes d'apprentissage que l'approche constructiviste.

Ces divergences et ressemblances ont fait l'objet de clarification et de négociation entre les partenaires de la recherche-action qui ont utilisé différents modes de fonctionnement, différents "séquençages $»^{7}$ (figures 1 à 4) qui tentaient de composer avec ces deux approches pour les activités de la formation concernées par une pédagogie active, mais non basées sur le développement d'un projet.

Le séquençage de la figure 1 a été utilisé pour travailler l'évaluation d'outils didactiques en ErE :

- analyse individuelle d'un outil et évaluation en 2 points positifs et 2 points négatifs ;

- identification, formulation et classification des critères d'évaluation en séance plénière ;

- comparaison avec 2 grilles d'évaluation faites par des chercheurs en ErE.

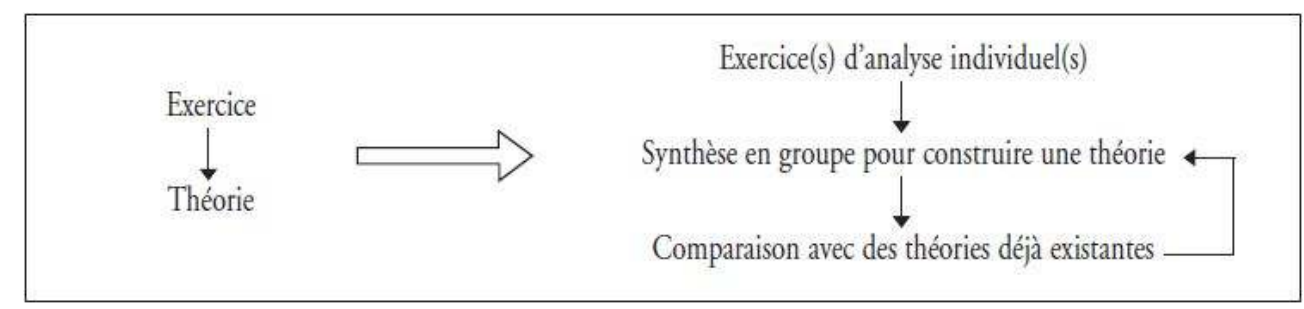

Figure 1 : Ce qu'est devenu le schéma inductif classique

Le séquençage de la figure 2 quant à lui a été utilisé pour travailler la définition de l'environnement :

- présentation de différentes définitions de l'environnement ;

- comparaison en équipe des définitions : points identiques, différents et importants ;

- construction en équipe d'une définition de l'environnement.

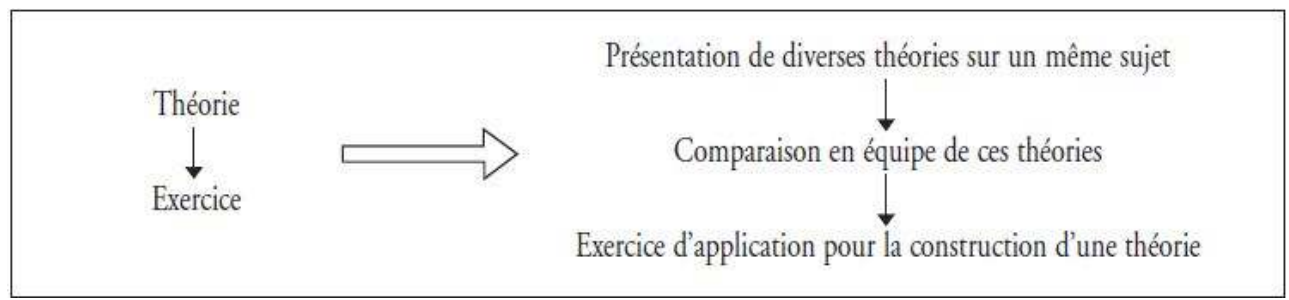

Figure 2 : Ce qu'est devenu le schéma déductif classique

Le séquençage de la figure 3 a été utilisé pour travailler la définition de l'ErE :

- présentation d'une définition de l'ErE et de la typologie de Lucas (1980-81) ;

- analyse d'activités d'ErE sur film vidéo à partir de la définition et de la typologie ;

- choix d'une activité d'ErE dans un répertoire d'activités conçues par des associations environnementales. 


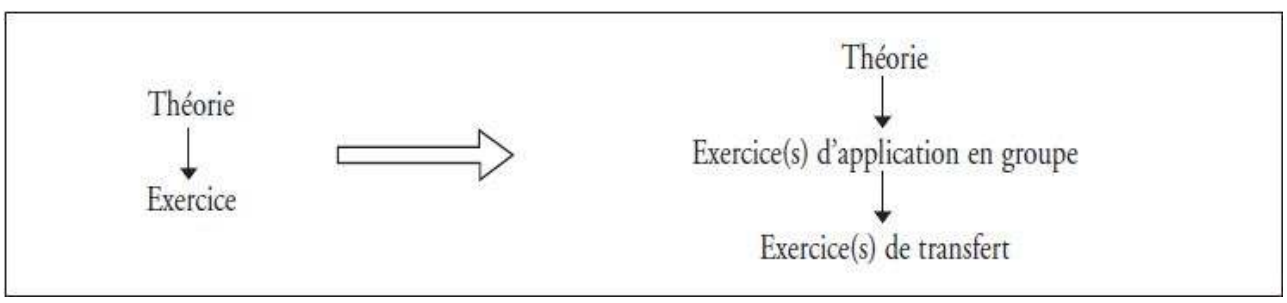

Figure 3 : Autre exemple de transformation du schéma déductif

Enfin, le plus important fut un schéma associant induction et déduction (figure 4).

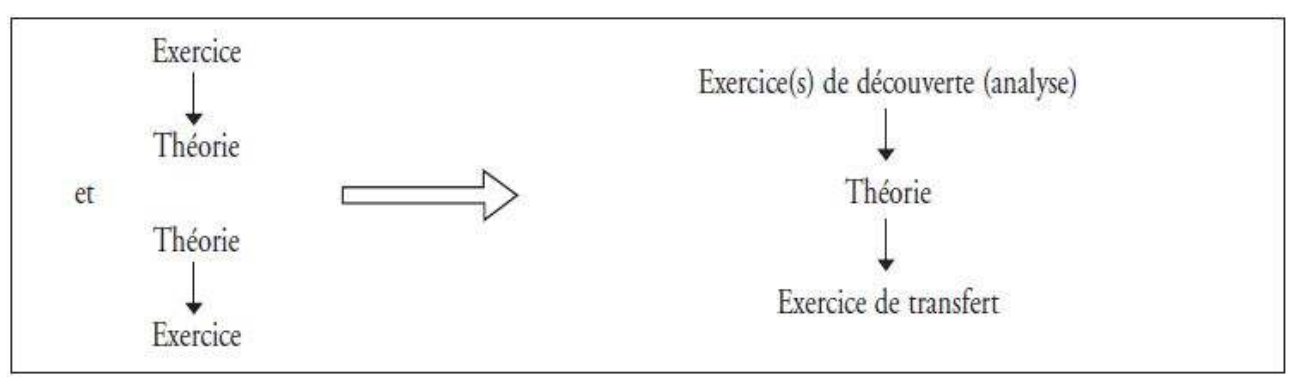

Figure 4 : Schéma associant induction et déduction

D'autres combinaisons étaient possibles. C'est ainsi que par la suite, une série d'activités sur un même thème a été construite qui utilisait à la fois le premier, le deuxième et le troisième séquençage présentés ci-dessus (figures 1 à 3).

L'existence d'activités non liées à l'approche résolutique dans la formation a donc provoqué un premier choc culturel qui a permis la création de divers « séquençages " négociés à l'intersection des conceptions socioprofessionnelles respectives. Comme on peut le voir dans ces "séquençages", il y a eu une interpénétration de l'approche constructiviste et de celle de l'induction/déduction pédagogique.

La prise en compte des différences de cultures socioprofessionnelles a permis l'analyse des présupposés respectifs, dans une démarche de réflexion critique. Cela a abouti à un développement de nouvelles stratégies de travail, plus qu'à une simple intersection de celles-ci. Ce développement se marque, entre autres, dans le fait que les professeurs en question ont réutilisé ces séquençages dans d'autres cours non liés à l'ErE qu'ils dispensent à l'ISP.

\section{Une deuxième " émergence »}

L'élément qui a permis, voire "précipité ", le choc culturel avec remise en question du modèle initial de formation, fut la question de la théorie à inclure dans le travail de gestion de projet pédagogique suite à un changement, une forte réduction, du temps imparti. En effet, lors d'une première expérimentation, la formation s'était déroulée en deux semaines de stage, une au tout début de l'année et une en fin d'année, entre lesquelles les étudiants devaient mener un projet de résolution de problème environnemental. Lors de la deuxième année d'expérimentation, la réforme des ISP entreprise par le ministre de l'Enseignement supérieur ne permettait plus d'allouer autant de temps aux stages : une seule semaine était disponible. 
Il fallait donc remodeler la formation pour atteindre le même type d'objectifs, mais sans pouvoir déployer une véritable pédagogie de projet (qui requiert du temps pour une démarche progressive et métacognitive). Pour pouvoir regagner du temps, il nous fallait introduire des moments plus théoriques et procéder à un travail moins basé sur le contact direct avec la réalité. Le débat et les négociations sur les liens qui unissent théorie et pratique, sur les notions d'application, de transfert, d'induction, de déduction et de constructivisme ont repris de plus belle, car il fallait reprendre le chapitre "démarches de la résolution de problème" sans pouvoir les pratiquer intégralement, mais toujours dans une optique de pédagogie active. De cette négociation est née un «séquençage didactique » nouveau. Ce « séquençage " (tel que vécu par l'élève) est assez complexe en réalité, mais il peut se résumer/simplifier provisoirement tel qu'indiqué dans la figure 5.

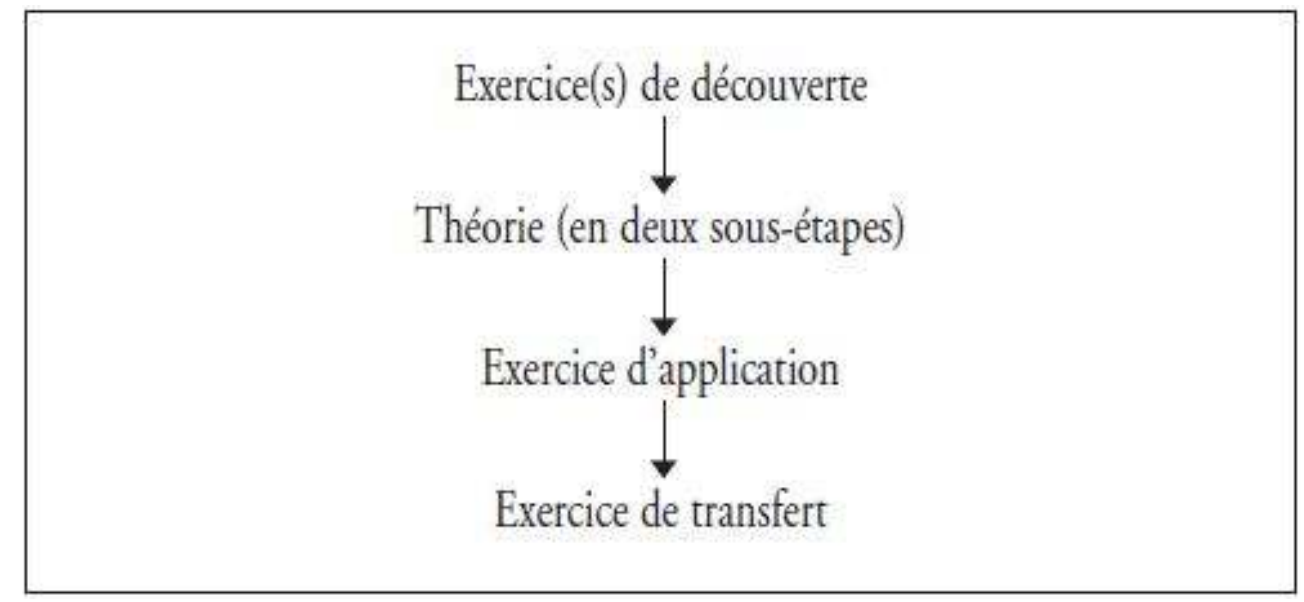

Figure 5 : Nouveau séquençage proposé

Pour pouvoir appliquer ce "séquençage " théorique à des objectifs et des contenus spécifiques, il a été établi une manière de "penser » et de " concevoir » les activités pour chacune des quatre étapes du séquençage. À cet effet, nous avons systématisé les diverses étapes de la démarche utilisée lors des réunions de préparation des activités de formation en ErE. Le même schéma didactique se compose donc de deux premières couches : une pour la façon de préparer le travail lors de la conception (figure 6) et une autre couche pour la façon de la faire vivre aux élèves en classe (voir figure 7).

Notons qu'un changement d'ordre dans les éléments du schéma est proposé. De plus, la pensée qui était au départ linéaire prend la forme d'une spirale quand on la met en action. Il nous est apparu aussi que parmi les professeurs qui collaboraient avec les enseignants engagés en ErE, certains manquaient de formation pédagogique en ce qui a trait aux travaux de groupe ou de synthèse collective par exemple, et que cela (associé aussi parfois au manque de temps) pouvait empêcher de se lancer dans ce type d'activités constructivistes. De ce fait, une version plus « douce » du cycle didactique a été conçue, version qui est plus "conductiviste» (tout en restant dans une vision «fonctionnelle » de l'apprentissage, Leclerq 1992). 


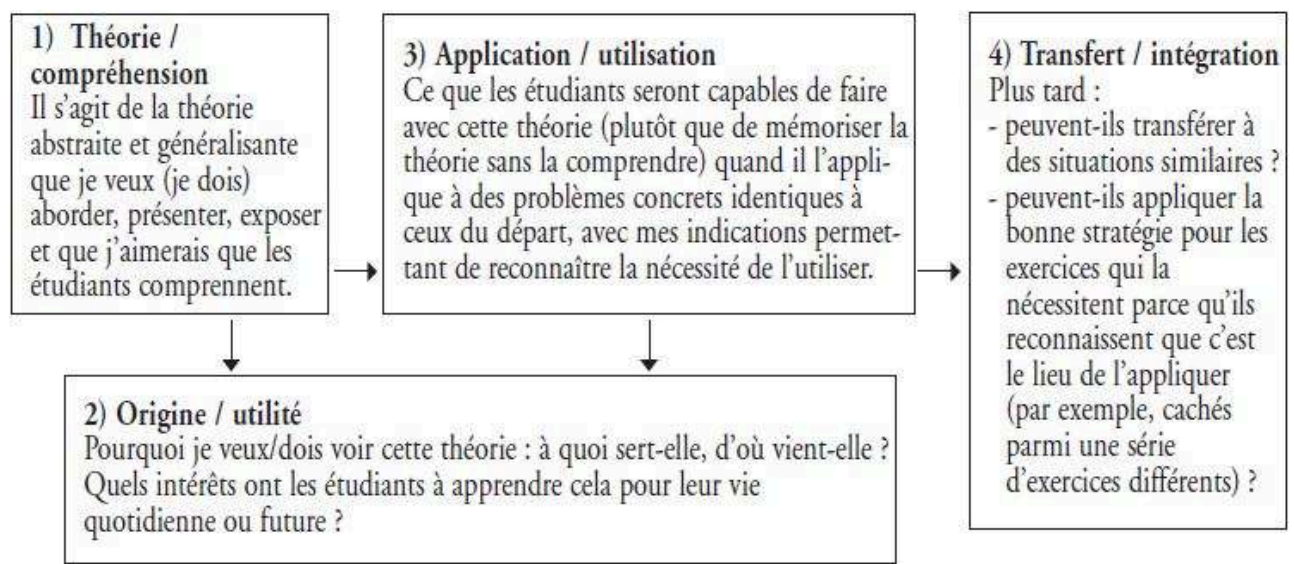

Figure 6 : Première « couche » du schéma (conception des activités) - Comment je " pense » mes activités quand je les prépare

\begin{tabular}{|c|c|c|}
\hline $\begin{array}{l}\text { 2b) Théorie générale } \\
\text { Que vais-je expliquer en plus } \\
\text { que les étudiants n'ont pas pu } \\
\text { retrouver à travers leurs expé- } \\
\text { riences et qui permet une géné- } \\
\text { ralisation plus grande ou un } \\
\text { niveau de profondeur plus } \\
\text { grand? } \\
\text { 2a) Théorie restreinte } \\
\text { Peut-on reconstruire (en } \\
\text { groupe, collectivement) la plus } \\
\text { grande partie possible de la } \\
\text { théorie à partir : } \\
\text { - d'observations de nos essais; } \\
\text { - de l'expérience d'autres } \\
\text { personnes; } \\
\text { - d'expériences passées } \\
\text { (observation réfléchie) } \\
\text { permettant in fine } \\
\text { l'abstraction? }\end{array}$ & $\begin{array}{l}\text { 3) Application / } \\
\text { utilisation } \\
\text { - Utiliser les } \\
\text { exercices initiaux } \\
\text { qui étaient trop } \\
\text { difficiles à } \\
\text { résoudre sans le } \\
\text { passage par la } \\
\text { théorie. } \\
\text { - Idem avec } \\
\text { d'autres exercices } \\
\text { identiques où } \\
\text { seules les } \\
\text { données } \\
\text { changent, par } \\
\text { exemple. }\end{array}$ & $\begin{array}{l}\text { 4) Transfert / intégration } \\
\text { Plus tard: } \\
\text { - proposer des exercices présentant des } \\
\text { situations proches (mais pas } \\
\text { identiques), par exemple où la façon } \\
\text { de présenter le problème change, mais } \\
\text { les éléments restent les mêmes; } \\
\text { - inclure des exercices qui utilisent cette } \\
\text { théorie au sein d'une série d'exercices } \\
\text { différents mélangés ou d'exercices } \\
\text { piégés (où il manque des données } \\
\text { permettant d'appliquer la procédure } \\
\text { ou qui sont absurdes) ; } \\
\text { - on peut aussi faire rechercher des } \\
\text { exercices similaires par les étudiants } \\
\text { ou faire construire et résoudre } \\
\text { mutuellement des exercices du type ; } \\
\text { - on peut aussi construire un exercice } \\
\text { complexe proche de la vie où cette } \\
\text { théorie est utile à une partie de la } \\
\text { résolution de l'exercice. }\end{array}$ \\
\hline & & \\
\hline \multicolumn{2}{|c|}{$\begin{array}{l}\text { 1) Origine/utilité } \\
\text { Partir d'un problème que la théorie explique ou solutionne } \\
\text { et demander aux étudiants de le résoudre (un défi) et les } \\
\text { laisser piétiner un peu (en équipe puisque cela permettra de } \\
\text { comparer les différences de point de vue). Cela créera un } \\
\text { choc qui légitimera l'apprentissage de la théorie. }\end{array}$} & \\
\hline
\end{tabular}

Figure 7 : Deuxième « couche » du schéma (activités en classe) - Comment j'« organise » l'apprentissage (dans une vision socioconstructiviste

Les éléments de cette version «conductiviste fonctionnelle» sont présentés dans le tableau 1. Ces éléments constituent la troisième couche du schéma, mais ils sont plus faciles à présenter sous forme de tableau. 
Tableau 1 : Troisième couche du schéma (activités en classe) - Comment je « vis » les activités (vision " conductiviste fonctionnelle »)

\begin{tabular}{|c|c|}
\hline Étapes & Activités de cette étape dans une vision de conductivité fonctionnelle \\
\hline 1) Origine / utilité & $\begin{array}{l}\text { Introduire le cours en donnant des exemples d'application dans le } \\
\text { quotidien familial, scolaire ou autres où cette théorie est utilisée. } \\
\text { Commencer par expliquer pourquoi et dans quel contexte la théorie a } \\
\text { été mise au point. Qu'est-ce qu'elle mettait en évidence, quelles } \\
\text { difficultés a-t-elle permis de surmonter? }\end{array}$ \\
\hline 2) Théorie / compréhension & $\begin{array}{l}\text { - lllustrer, décomposer, simplifier, schématiser la théorie (d'après ce que } \\
\text { les étudiants de l'année dernière ont vécu comme difficultés ou d'après } \\
\text { ce que ceux-ci n'ont pas compris lors de mon premier essai). }\end{array}$ \\
\hline 3) Application / utilisation & $\begin{array}{l}\text { Il ne s'agit pas seulement de rendre le cours théorique un peu plus } \\
\text { ludique ou attrayant mais de proposer après la théorie des exercices } \\
\text { individuels où l'on applique celle-ci, exercices proches des exemples } \\
\text { initiaux ou de ceux que j'ai pris pour expliciter la théorie. } \\
\text { Dans ce cas, seules les données changent, le type de présentation de la } \\
\text { question reste la même que lors de la théorie. } \\
\text { Avec l'expérience, on peut construire une série d'exercices qui mettent } \\
\text { chacun en avant-plan un aspect de la théorie que les étudiants ont de } \\
\text { la difficulté à comprendre. }\end{array}$ \\
\hline 4) Transfert / intégration & $\begin{array}{l}\text { Plus tard: } \\
\text { Proposer des exercices individuels présentant des situations proches } \\
\text { (mais pas identiques), par exemple où la façon de présenter le } \\
\text { problème change, mais les éléments restent les mêmes. } \\
\text { Inclure des exercices qui utilisent cette théorie au sein d'une série } \\
\text { d'exercices différents et mélangés ou au sein d'exercices piégés (où il } \\
\text { manque des données permettant d'appliquer la procédure ou qui sont } \\
\text { absurdes). }\end{array}$ \\
\hline
\end{tabular}

\section{Comparaison avec le modèle de l'apprentissage expérientiel de Kolb}

La rencontre au sein du projet européen Commenius avec des chercheurs de l'UAB (Espagne), Rosa-Maria Pujol et Rosa-Maria Tarin (2002) qui travaillent à l'amélioration de l'approche « expérientielle » de Kolb (1971) au moyen de l'évaluation / régulation, nous a fait comprendre la portée de ce « séquençage didactique ». Il n'est pas seulement une innovation par rapport au niveau initial et respectif de notre équipe, mais une proposition susceptible d'intéresser les didacticiens en ErE comme nous montre la comparaison avec les éléments de la figure 8.

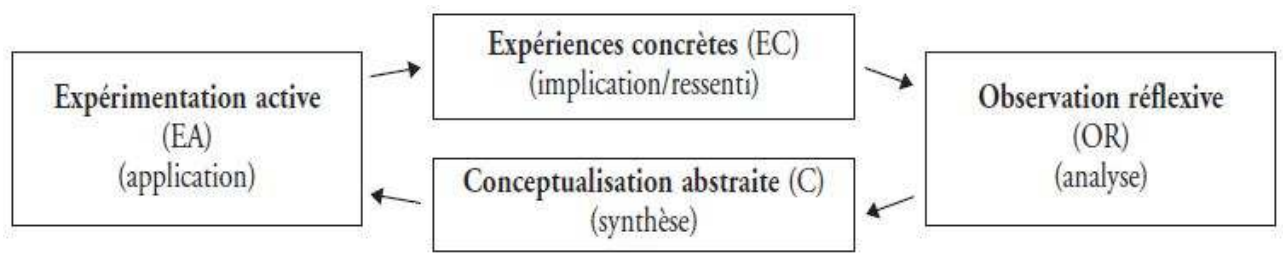

Figure 8 : Représentation graphique du modèle d'apprentissage « expérientiel » de Kolb (1971)

Kolb propose un modèle d'apprentissage dit «expérientiel ». Si l'on résume le point de vue de cet auteur, l'apprentissage sur base d'expériences concrètes est un processus constitué de 4 phases qui forment un cycle. Toutes les expériences d'apprentissage de l'individu se déplacent le long de ce cycle. Dans un premier temps, la personne en situation d'apprentissage ou de résolution de problèmes vit elle-même une situation 
d'expérience, c'est l'expérimentation concrète: le ressenti. Elle est suivie par l'observation réflexive qui consiste à faire des observations sur l'expérience vécue et à réfléchir à leur signification. Cette étape conduit à la formation de concepts et à la formulation de généralisations qui intègrent les observations et réflexions, c'est la conceptualisation abstraite. Vient ensuite l'expérimentation active où le sujet vérifie les hypothèses alors générées dans de nouvelles situations concrètes.

En fait, la première étape de notre deuxième « couche » du schéma (« origine/utilité ») correspond à l'étape des "expériences concrètes» dans le modèle de Kolb (1971), l'étape 2a ("théorie restreinte ») correspond plutôt à l'étape " observation réflexive ", l'étape $2 b$ («théorie générale») quant à elle correspond plutôt à l'étape « conceptualisation abstraite ». Par contre, l'étape « expérimentation active » de Kolb (1971) se trouve subdivisée dans notre schéma en deux étapes qui souligne la différence entre application et transfert d'une théorie telle qu'explicitée dans les figures 6 et 7.

Le « séquençage » didactique ainsi créé par l'équipe a l'avantage de :

- mieux mettre en évidence la différence entre application et transfert que le modèle de Kolb (1971), ce qui est capital en ErE puisque les acquis du travail en classe devraient se répercuter en dehors de celle-ci ;

- souligner la différence existante entre les activités vécues par les étudiants et la façon dont le professeur pense ses activités lorsqu'il les prépare. Le schéma de la figure 6 constitue un guide permettant au professeur de passer de l'idée à l'activité en suivant un modèle de pensée et non pas en appliquant une « recette ». Le schéma de la figure 7 montre ce que les étudiants vont vivre réellement, ce qui ne suit pas le même processus que la conception ;

- permettre au professeur d'acquérir peu à peu le séquençage didactique ou de s'adapter à un manque de temps (le temps prévu étant fonction d'une approche conductiviste / transmissive).

En outre, un tel schéma peut permettre la mise en place d'une évaluation formative et formatrice tout au long de l'apprentissage, à chacune des étapes. En effet, dans un tel processus, il est difficile d'imaginer des interrogations sommatives à la fin de chacune des trois premières étapes puisqu'il s'agit d'un processus global. En nous inspirant partiellement des travaux de Pujol et Tarin (2002), nous proposons les éléments d'évaluation présentés au tableau 2. Ces éléments constituent la quatrième couche du schéma, mais ils sont plus faciles à présenter sous forme de tableau. 
Tableau 2 : Quatrième « couche » du schéma (évaluation) - Où (quand) évaluer dans cette spirale didactique (et justification)

\begin{tabular}{|c|c|c|c|c|}
\hline Étapes & \multicolumn{3}{|l|}{ Type d'évaluation } & Justification \\
\hline \multirow[t]{2}{*}{$\begin{array}{l}\text { 1) Origine / } \\
\text { utilité }\end{array}$} & \multicolumn{3}{|c|}{$\begin{array}{l}\text { Pré-test (cela vaut-il la peine de travailler cela ; les étudiants ne le } \\
\text { savent-ils pas déjà ?) }\end{array}$} & \multirow{2}{*}{$\begin{array}{l}\text { Absence de ce pré-test, 'e'est } \\
\text { faire l'hypothèse que les } \\
\text { étudiants ne savent rien } \\
\text { (tabula rasa). }\end{array}$} \\
\hline & \begin{tabular}{|l} 
Dans une vision inductive \\
fonctionnelle: \\
Demander aux étudiants sils \\
savent à quoi sert cette théori \\
(formuler des hypothèses et \\
expliquer à quoi cette théorie \\
leur sera utile).
\end{tabular} & & $\begin{array}{l}\text { une vision socio- } \\
\text { uctiviste: } \\
\text { er les élèves pendant les } \\
\text { (différentes visions) et } \\
\text { e en place une auto- } \\
\text { tion à la fin de l'essai afin } \\
\text { lir le constat de " manque ". }\end{array}$ & \\
\hline \multirow[t]{2}{*}{$\begin{array}{l}\text { 2) Théoriel } \\
\text { compréhension }\end{array}$} & \multicolumn{3}{|c|}{$\begin{array}{l}\text { Évaluation formative (sans inclure cette note dans la cotation } \\
\text { finale) et déclarative (ÉFD) }\end{array}$} & \multirow{2}{*}{$\begin{array}{l}\text { Absence de l'ÉFD, c'est faire } \\
\text { l'hypothèse que les étudiants } \\
\text { avaient compris le cours } \\
\text { théorique. }\end{array}$} \\
\hline & \multicolumn{2}{|c|}{$\begin{array}{l}\text { Vision inductive fonctionnelle: } \\
\text { (1) la restitution de mémoire, } \\
\text { (2) les discours explicatifs des } \\
\text { (étudiants, } \\
\text { (3) la compréhension à travers des } \\
\text { questions subtiles sur la théorie ou } \\
\text { des exemples à fournir ou à juger. }\end{array}$} & $\begin{array}{l}\text { Vision socio- } \\
\text { Constructiviste: } \\
\text { Faire trouver des exemples } \\
\text { de la théorie sur les cas qui } \\
\text { ont servit à la construire. }\end{array}$ & \\
\hline \multirow[t]{2}{*}{$\begin{array}{l}\text { 3) Application / } \\
\text { utilisation }\end{array}$} & \multicolumn{3}{|c|}{$\begin{array}{l}\text { Évaluation formative (sans inclure cette note dans la cotation finale) } \\
\text { et formatrice (que lélève se rende compte lui-mếme de son niveau } \\
\text { dans lutuilisation de la théorie) et donc surtout evaluation diagnos- } \\
\text { tique (ÉFFD) (regarder le fonctionnement pas seulement le résultat) }\end{array}$} & \multirow{2}{*}{$\begin{array}{l}\text { Absence de l'ÉFFD, c’est } \\
\text { faire l'hypothèse que les } \\
\text { étudiants avaient déjà tout } \\
\text { compris au cours théorique } \\
\text { et qưils sont déjà aptes à } \\
\text { l'appliquer. }\end{array}$} \\
\hline & $\begin{array}{l}\text { Vision inductive } \\
\text { fonctionnelle: } \\
\text { Les hypothèses et l'utilité } \\
\text { supposées par les étudiants } \\
\text { se montrent-elles vraies? }\end{array}$ & $\begin{array}{l}\text { Vision s } \\
\text { (Auto-) } \\
\text { arrivent } \\
\text { exercice } \\
\text { similaire }\end{array}$ & $\begin{array}{l}\text { cio-constructiviste: } \\
\text { valuer si les étudiants } \\
\text { appliquer la théorie sur les } \\
\text { initiaux ou des exercices }\end{array}$ & \\
\hline $\begin{array}{l}\text { 4) Transfert / } \\
\text { intégration }\end{array}$ & \multicolumn{3}{|c|}{$\begin{array}{l}\text { ÉFFD2 : Idem que précédemment mais sur des situations de } \\
\text { transfert. Cette étape constitue déja une forme de test du niveau } \\
\text { précédent, mais elle ne peut être vraiment considérée comme léva- } \\
\text { luation finale car les aspects novateurs de la situation "transfert " } \\
\text { linterdisent. }\end{array}$} & $\begin{array}{l}\text { Absence de l'ÉFFD2, c'est } \\
\text { faire l'hypothése que les } \\
\text { étudiants sont aussi capables } \\
\text { sans le professeur quavec } \\
\text { celui-ci. }\end{array}$ \\
\hline 5) Fin de cycle & \multicolumn{3}{|c|}{$\begin{array}{l}\text { Évaluation sommative : Post-test équivalent à la phase } 4 \text { mais plus } \\
\text { tard encore (x mois). }\end{array}$} & $\begin{array}{l}\text { Absence d'évaluation } \\
\text { sommative, c'est faire l'hypo- } \\
\text { thèse que la compétence est } \\
\text { acquise à long terme. }\end{array}$ \\
\hline
\end{tabular}

\section{Planifier la prise en compte des émergences ?}

Afin de se rendre capable d'utiliser les émergences pour l'auto-développement mutuel et donc de dépasser la vision positiviste de la sérendipité, il est nécessaire de construire des démarches de fonctionnement de l'équipe et de réfléchir au rôle du chercheur dans ce cadre-là.

Ci-dessous sont présentées les stratégies que nous avons mises en place. Nous parlerons dorénavant sur le mode du "nous » et du «je », car elles n'ont pas été validées dans d'autres contextes et sont le fruit de l'histoire particulière de la recherche-action à la base de cet article. De ce fait, les limites de ces stratégies seront soulignées.

\section{Les stratégies mises en place par l'équipe de cette recherche-action}

La difficulté principale venait du fait de réussir à échanger nos idées sans se heurter et se vexer les uns les autres lors des réunions de préparation ou lors des réunions d'évaluation. Notre équipe s'est efforcée d'installer et de renforcer un climat de libreéchange d'idées basé sur la confiance dans la plus grande efficacité du travail collectif et sur le respect de la parole lors des réunions. Si cela est un objectif souvent prescrit pour ce type de réunions, il est plus rare que l'on propose une stratégie concrète qui permette de construire une réunion rendant possible ce type d'échange. Peu à peu, les 
membres de notre équipe ont adopté une démarche en trois étapes doublées « d'habitudes langagières » et de rétroactions :

1. Quand une idée d'activité doit être trouvée ou un problème doit être résolu, celui qui a une solution/idée ne donne pas la solution/idée, mais propose une solution/idée à débattre, de manière " dépersonnalisée ». Il doit être écouté jusqu'au bout sans être interrompu.

Nous avions pris l'habitude langagière, de dire : " voici une proposition à casser... » et d'éviter des phrases telles que «j'ai la solution, voilà comment il faut faire... ».

1. Celui qui parle sait à l'avance que le rôle des autres formateurs est de vérifier si sa solution/ idée ne comporte pas d'effets pervers par rapport à la situation concrète où elle doit être appliquée, surtout s'il s'agit d'un transfert d'une autre situation, mais cela se fera sans l'attaquer personnellement. La critique positive ou négative doit concerner la proposition/ solution et non pas la personne et doit être justifiée, et ce, sur un mode personnel, en tant qu'opinion. En outre, il n'est pas question à ce moment-là de proposer autre chose à la place pour ne pas créer le conflit. Nous avions pris l'habitude langagière, de dire: «je trouve personnellement que les points positifs/négatifs de cette proposition/solution sont... parce que, par rapport à ma vision... » et d'éviter des phrases telles que « ta proposition n'est pas bonne parce que..., voilà comment il faudrait faire... ».

2. Ensuite, le rôle de l'équipe est d'améliorer tous ensemble cette solution/idée afin d'en construire une autre qui n'aurait pas les mêmes défauts. Si cela ne fonctionne pas, on recommence à l'étape 1 .

Nous avions pris l'habitude langagière de dire « une version améliorée de cette proposition serait...».

Dans le cas où l'on avait appliqué plus d'une fois le mécanisme, on possédait plusieurs solutions avec leurs critiques, de ce fait on tentait alors d'intégrer les solutions. Nous avions pris l'habitude langagière de dire «si on mélange ces solutions, nous pourrions faire ceci...».

Avec le temps, quand le climat de libre-échange fut vraiment bien installé, nous avons accéléré la procédure en permettant de proposer plusieurs idées/solutions dès la première étape et en appliquant les critiques de la deuxième étape à chacune d'entre elles. Même dans ce cadre-là, les habitudes de formulation langagière sont restées.

41 C'est lors de la critique des idées/solutions que se clarifiaient les conceptions de chacun. C'est ainsi que se négociait non pas seulement un point d'intersection admissible entre les deux cultures socioprofessionnelles, mais la reconstruction d'une vision nouvelle qui englobe les points de vue initiaux. Concrètement, cette stratégie de travail nous permettait :

- d'éviter qu'il y ait des atteintes personnelles en cas de critiques;

- d'éviter que quelqu'un s'attache à défendre, envers et contre tous, sa solution/idée contre celle d'un autre ;

- d'éviter de tomber dans le piège de la pensée unique ;

- d'anticiper les risques potentiels, dans une pensée systémique.

Cependant, cette procédure avait ces limites. Il y avait parfois des dérapages. Avec le temps, nous nous sommes rendu compte que cela arrivait principalement lors de la recherche de solutions à un problème qui semblait, intuitivement, hors de notre zone de pouvoir. Nous avons tenté de changer notre fonctionnement affectivo-cognitif en adoptant une attitude en deux temps face à ces problèmes apparemment hors zone de 
pouvoir. Il s'agit de se rendre capable de transformer les réalités du terrain plutôt que d'expliquer tout en termes de " fautes » ou de manques antérieurs.

1. Ne plus réfléchir en termes de "fautes" (celle des étudiants, celles des personnes extérieures, celles de l'un d'entre nous, celle de l'équipe, etc.), de "coupables » selon le mode culturel du « péché » de la civilisation judéo-chrétienne dont nous sommes issus, mais de constater les difficultés, sans personnaliser ni n'accuser personne.

Nous avions pris l'habitude langagière de ne plus dire " c'est la faute de... ", mais plutôt " un des éléments qui explique le problème, c'est... ».

1. Réfléchir en termes de mesures à prendre préventivement comme « conditions nécessaires, quoique peut-être pas suffisantes, pour enrayer le phénomène, le problème » : quelles sont les solutions, les mesures que nous, les formateurs, pouvons prendre à l'avance pour diminuer le risque que de telles difficultés réapparaissent ? Par exemple, la première année, certains étudiants arrivaient en retard (ou pas du tout) à la formation qui était placée en octobre (par le fait d'inscription tardive). La deuxième année, nous l'avons placée au mois de mars. Cela ne veut pas dire qu'il n'y a plus d'absences, mais elles ont fortement diminué.

Nous avions pris l'habitude langagière de dire « cela n'arrange pas tout, mais tel élément pourrait résoudre, au moins partiellement, le problème ».

1. Dans la suite de la recherche, ces procédures de discussion lors des réunions entre formateurs ont dû être complétées aussi par des procédures lors des activités afin que le respect mutuel se prolonge aussi en classe, en face des étudiants.

Ainsi, premièrement, par rapport à la possibilité d'interventions verbales d'un professeur par rapport à un autre, lorsqu'on donnait cours, nous avons d'abord conçu l'accord qui suit : même si les activités ont été créées en commun, chaque activité est gérée par un seul professeur (« le titulaire»), les autres observent, préparent le matériel, etc., mais silencieusement. Ils offrent un appui. De sorte que le « titulaire » n'est pas perpétuellement dérangé par des interventions intempestives. Puis, peu à peu, quand la confiance s'est installée, un deuxième mécanisme a été ajouté. Les «professeurs-accompagnants » ont le droit d'intervenir pendant le cours selon trois cas précis :

- soit le «professeur-titulaire » à la fin d'un chapitre ou sur un point délicat demande si les autres veulent intervenir,

- soit un des «professeurs-accompagnants » «lève son doigt » pour obtenir la possibilité d'intervenir,

- soit le titulaire ne s'en sort plus et profite d'un travail de groupe ou d'une pause pour demander à un autre de prendre le relais.

Deuxièmement, comme il arrivait que des demandes imprévues viennent de la part des étudiants pendant les activités (lors des travaux de groupe principalement, mais aussi en séance plénière), nous avons adopté les deux stratégies suivantes :

- prévoir des garde-fous aux adaptations éventuelles du cours par le titulaire, engendrées par les cas particuliers; les objectifs et les consignes des activités ainsi que les critères d'évaluation doivent être clairs, concertés et toujours sous la main du responsable de la formation (ils doivent figurer dans la fiche didactique de l'activité) ;

- si un formateur se trouve confronté à une question non prévue (que ce soit sur le fond ou sur la forme), qui ne se résout pas en fonction des critères préétablis, il dit, tout simplement, aux étudiants que le cas nécessite un «appel à l'équipe» (question de fond) ou «une réponse collégiale » (question de forme).

De cette manière, premièrement, nous évitons de donner des informations contradictoires. Deuxièmement, nous montrons aux étudiants qu'il est possible de 
travailler en équipe de professeurs si l'on met en place les mécanismes nécessaires. Troisièmement, nous enrayons cette croyance qu'un professeur doit tout connaître et ne peut jamais avouer l'ignorance de quelque chose. Au niveau langagier, nous employons aussi : « je ne connais pas la réponse, mais je sais comment on la trouve », « on va chercher ensemble » ou « je te fournirais la réponse demain ».

\section{Les stratégies mises en place par le chercheur}

Le rôle du chercheur que j'ai adopté à ce niveau fut double :

- Repérer et favoriser les modes de fonctionnement (tels ceux expliqués ci-dessus) qui favorisent l'expression et le respect des différences de cultures socioprofessionnelles et si possible les modéliser afin d'en systématiser l'utilisation consciente par l'équipe. Par exemple, l'expression " voici une proposition à casser » avait été utilisée par une personneressource, lors d'une réunion de préparation. À la réunion suivante, j'ai pris l'habitude de présenter toutes mes propres propositions en commençant par la même phrase. À la réunion d'après, j'ai lancé la discussion sur un ton joyeux par un « qui aurait une proposition à casser » et ainsi de suite.

- Repérer et garder la trace des différences et des ressemblances de visions provenant des cultures socioprofessionnelles qui ont été exprimées ainsi que la trace des visions communes qui se sont construites, tout au long du travail.

\section{Observations sur les stratégies utilisées}

Toutes les stratégies présentées ci-dessus sont le fruit de l'histoire d'une équipe et de la capacité que nous avons eu à garder les formulations langagières qui reflétaient notre façon de voir la chose. L'emploi du «nous » ou du «je » dans ces propositions n'est pas anodin. C'était le fruit d'une pratique qui n'a pas encore pu être reproduite et testée dans d'autres contextes. Au lecteur, d'en évaluer la possibilité de transfert et d'adaptation à son propre contexte.

Nous pensons que le transfert et l'adaptation à votre contexte nécessiteront une recherche dans votre équipe de formulations langagières qui devront aussi être représentatives de votre façon de voir la gestion d'une réunion. Cette dernière doit être elle-même clarifiée. En fait, c'est le principe qui doit être adopté et pas spécialement notre façon de dire ou de faire. Ce principe comporte 4 points :

- construction d'une vision de réunion (réunion de type « climat d'échange d'idées basé sur la confiance en une plus grande efficacité systémique du travail collectif », par exemple);

- identification des situations où ce climat doit fonctionner (en réunion de préparations, d'évaluation, lors des cours, par exemple);

- construction/modélisation de procédures adaptées à ces différentes situations ;

- adoption d'habitudes langagières qui reflètent votre façon de penser.

D'autres observations sur les stratégies utilisées méritent d'être soulignées :

- notre équipe avait déjà pris conscience qu'il existait des différences de culture socioprofessionnelle entre le chercheur et les enseignants; nous avions déjà travaillé ensemble auparavant, mais il est vrai que les stratégies utilisées les ont encore plus mises en évidence ;

- les démarches expliquées précédemment et la discussion ont permis à chacun de connaître plus précisément la vision de l'autre ; 
- les démarches mises en place ont constitué notre savoir-faire, notre habilité à mettre en jeu nos différentes représentations et à en tirer profit ;

- la stratégie de repérage et de renforcement de ce mode de fonctionnement déployée par le chercheur a, en fait, correspondu à des savoir-faire stratégiques (Leclerq, 1992), soit des savoir-faire qui permettent de s'améliorer soi-même.

Il faut reconnaître que, dans notre cas, l'attitude d'ouverture à la culture socioprofessionnelle de l'autre existait au préalable pour les membres de notre équipe, par les parcours individuels respectifs. Les stratégies présentées n'ont pas créé cette attitude. À la limite, on peut estimer qu'elles l'ont renforcée. Or, il est important de souligner que dans notre situation, il n'y avait pas de conflit de pouvoir entre le chercheur et les enseignants. L'absence de conflit de pouvoir est un facteur qui permet plus facilement l'adoption d'une attitude de respect. Nous ne savons donc pas si dans un contexte de lutte de pouvoir, ce type de stratégies peut se mettre en place.

\section{Conclusion}

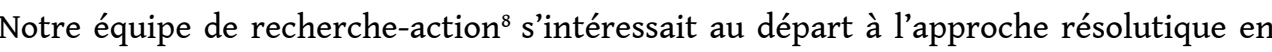
pédagogie de projets pour le volet "Action » et aux facteurs adjuvants ou opposants à la formation initiale en ErE dans les ISP pour le volet « Recherche ». Les émergences ont débouché d'un côté sur une formulation conjointe de nouveaux "séquençages " didactiques et d'une approche didactique globale (proche de l'approche « expérientielle » de Kolb, 1971) grâce à la clarification et la négociation des modes de fonctionnement hérités des sous-cultures socioprofessionnelles à travers des formulations langagières convenues et représentatives de cette volonté de clarifier et de négocier qui a abouti, in fine, à l'auto-développement mutuel des parties.

D'un autre côté, ce travail de terrain a donné lieu à un (autre) changement de conception sur le statut même des émergences en recherche-action. Dans une vision de critique sociale, les émergences ne viendraient pas seulement de la découverte heureuse et inattendue (sérendipité), mais du choc (sous-) culturel lui-même, à travers la clarification et la renégociation que les partenaires de recherche voudront bien mener. Il ne suffit donc plus de dire « attention en recherche-action, il peut y avoir des émergences, soyez ouverts ", mais il faut attirer l'attention sur le fait que ces émergences seront souvent le fruit même des différences culturelles. Un choc culturel a d'autant plus de chance de se mettre en place si les partenaires ne cherchent pas seulement à se trouver des intérêts respectifs à un travail en commun (Piette et al., 2000), mais s'ils essayent de se construire une vision commune nouvelle qui les fait évoluer à travers la mise en place de modes de fonctionnement appropriés.

De ce fait, une première esquisse du rôle possible que peut adopter un chercheur lors d'une recherche-action concernant la prise en compte des cultures socioprofessionnelles aboutit d'un côté à proposer, repérer, favoriser et modéliser les modes de fonctionnement en équipe respectueux de ces différences socioprofessionnelles puis de l'autre côté, à repérer les différences et les ressemblances de visions ente les protagonistes, ainsi que les tentatives de reconstruction d'une vision commune.

$51 \mathrm{Si}$ ces derniers éléments n'ont pas le statut de "vérité scientifique », car intrinsèquement liés à une seule recherche, ils soulignent cependant le fait que le rôle 
du chercheur et le fonctionnement de l'équipe doivent faire l'objet d'un véritable travail de réflexion des parties prenantes afin de favoriser l'augmentation de la prise en compte des émergences d'origine sous-culturelle de type socioprofessionnel dans le cadre de la recherche-action.

Ce faisant, il s'agit simplement d'appliquer de manière consciente et active lors des partenariats en recherche-action, l'un des principes de l'approche critique selon Brookfield (1991) : «reconnaitre l'importance capitale du contexte (en particulier socioculturel) comme facteur d'explication d'une situation, d'un phénomène » (in Sauvé, 1994, p. 135).

\section{BIBLIOGRAPHIE}

Brookfield, S.D. (1991). Developing critical thinkers. San Francisco : Jossey-Bass Publishers. D’Hertefelt, M. (1992). Anthropologie culturelle : évolution, histoire, structure, fonction. Belgique : Presse Universitaire de Liège.

Kolb, D.A. (1971). Individual learning styles and the learning process. Cambridge (MA) : M.I.T. Sloan School.

Leclercq, D. (1992). Introduction à la technologie de l'éducation. Syllabus. Faculté de Psychologie et des Sciences de l'Éducation (FAPSE) : Université de Liège.

Lucas, A.M. (1980-81). The role of science education in education for the environment. Journal of Environmental Education, 12(2), 32-37.

Parot, F. and Richelle, M., (1992). Introduction à la psychologie : Histoires et méthodes. Paris : Presse universitaire de France.

Piette, S.-A., Legrand. E., Croizer, C., Goffin, L., von Frenckell, M., Philippet, C. et Wattecamps, J.M. (2000) Le partenariat au service de l'évaluation de projets d'éducation relative à

l'environnement. Éducation relative à l'environnement : Regards, Recherches, Réflexions, 2, 119-145.

Pujol, M.R. et Tarin, R.M. (2002) Diseño de una unidad didáctica. Communication non publiée présentée au Séminaire Des déchets scolaires à l'école à l'éco-citoyenneté, Bruxelles, 2002.

Sauvé, L. (1994). Pour une éducation relative à l'environnement : Éléments de design pédagogique. Montréal : Guérin.

\section{NOTES}

1. D’Hertefelt (1992) distingue deux manières de définir le terme culture. La première se situe au niveau formel: "c'est un phénomène d'un ordre particulier, non biologique, social, appris, largement inconscient» (p. 229). La seconde se situe au niveau du contenu dont il distingue quatre aspects : 1) un aspect matériel (outils, biens), 2) un aspect social (rapports sociaux), 3) un aspect normatif (règles de vie en commun, la morale), 4) un aspect symbolique (les idées et les diverses formes de représentation qui expriment le sens que les membres d'une société donnent 
à celle-ci, au monde et à l'existence humaine). C'est cette quatrième partie qui nous intéresse le plus.

2. «La standardisation des comportements (des objets, des idées) n'est jamais absolue. La culture n'emprisonne pas l'homme dans un carcan rigide au point de n'admettre aucune variation. Les anthropologues expriment cette idée en disant que la culture est proposée plutôt qu'imposée » (D’Hertefelt, 1992, p. 226).

3. « Les anthropologues désignent le processus d'apprentissage de la culture par deux termes qui se rapportent à la même réalité, mais sous des aspects différents : socialisation et enculturation » (D’Hertefelt, 1992, p. 226).

4. Le mot francisé « sérendipité » est reconnu par Parot et Richelle (1992).

5. Un Institut supérieur pédagogique est un institut non universitaire de formation des instituteurs et professeurs du secondaire inférieur $(B A C+3)$, anciennement appelé "école normale ».

6. Tout comme pour les professeurs retraduisant inconsciemment des modes de fonctionnement issus de la sous-culture scientifico-mathématique "proposée » lors de leur formation initiale, le pédagogue reprenait le principe du constructivisme "proposé» dans le cadre de sa propre formation en psychologie de l'apprentissage. Or toutes les pédagogies actives ne sont pas forcément basées sur le constructivisme, certaines pédagogies actives sont d'ailleurs antérieures aux travaux de Piaget, à la base du constructivisme.

7. Les schémas qui suivent n'illustrent pas le choc de culture socioprofessionnel, mais un exemple de ce que ce choc a produit comme nouveaux modèles dans le contexte de cette recherche-action.

8. Cette recherche-action peut être interprétée également comme une recherche-développement collaborative.

\section{RÉSUMÉS}

L'article explore la dimension culturelle de la formation en éducation relative à l'environnement dans le cadre de recherches-actions. Lors de celles-ci, les protagonistes sont souvent issus de sous-cultures socioprofessionnelles différentes impliquant des modes de fonctionnement différents. La prise en compte de ces différences culturelles est susceptible de poser la question fondamentale du statut des "émergences » en recherche-action : peut-on continuer à considérer que les éventuelles «émergences » susceptibles de s'y produire soient toutes le fruit du hasard, selon l'interprétation d'une science positiviste? Pour illustrer ce propos, un exemple concret d'émergence ayant son origine dans les différences de cultures en présence dans une rechercheaction en situation de formation sera présenté; des pistes permettant de tenir compte activement de ces émergences seront identifiées.

This paper explores the cultural dimension of environmental education training in the context of action-researches. In such researches, the actors usually come from different socio-professional cultures involving different ways of functioning. Taking into account these cultural differences can enable the exploration of the status of « emergences » in action-researches : can we continue to consider the probable « emergences » in action-research as coincidences, as suggested by the positivist vision of research? To illustrate this idea, an example of emergence in action-research 
resulting from cultural differences is presented and possibilities for actively taking into account such emergences are developed.

\section{INDEX}

Keywords : action research, environmental education, serendipity, culture

Mots-clés : recherche action, éducation relative à l'environnement, sérendipité, culture

\section{AUTEUR}

\section{EMMANUEL LEGRAND}

Psychologue et pédagogue de formation, il termine un doctorat en didactique des sciences de l'environnement à la Fondation Universitaire Luxembourgeoise. Il est chargé de recherches concernant l'éducation relative à l'environnement (ErE) et le milieu scolaire, principalement en formation initiale ou continue de professeurs. Il est également membre du jury de la médiathèque de Belgique pour la collection ErE et l'auteur avec Sylvie-Anne Piette d'un guide méthodologique de conception et d'évaluation d'un outil didactique en ErE. 